\title{
Solutions to Some Open Problems from Slaney
}

\author{
Branden Fitelson \\ Department of Philosophy \& Religion, Northeastern University.
}

\begin{abstract}
In response to a paper by Harris \& Fitelson [1], Slaney [6] states several open questions concerning possible strategies for proving distributivity in a wide class of positive sentential logics. In this note, I provide answers to all of Slaney's open questions. The result is a better understanding of the class of positive logics in which distributivity holds.
\end{abstract}

\section{Introduction}

Harris \& Fitelson [1] used Otter to prove distributivity in $\mathrm{E}_{\aleph_{0}}$ and other nonclassical sentential logics. Their proofs involved axiomatizations in terms of implication $(\rightarrow)$ and negation $(\neg)$. Slaney [6] showed how to prove these results in the positive fragments of these logics, which involve only implication $(\rightarrow)$, conjunction $(\wedge)$, and disjunction $(\vee)$. Slaney also provided a much more general framework for thinking about distributivity in a wide class of positive logics. This led him to state several open questions regarding strategies for establishing distributivity in this broad class of non-classical (positive) logics. In this note, I will provide answers to all of Slaney's open questions. All of these results were obtained using (various) automated reasoning tools 1

\footnotetext{
${ }^{1}$ I used a combination of prover9 [3], Otter [4, E [5], and Vampire 2] to solve Slaney's open problems. All proofs are presented in the APPENDIX, in Otter format.
}

Australasian Journal of Logic (13:4) 2016, Article no. 1 


\section{Slaney's Three (Background) Positive Log- ics}

Slaney [6] presents a large class of (positive) logics, which involve various combinations of the following axioms and rules (i.e., axiom and rule schemata) ${ }^{2}$

$\begin{array}{ll}(\mathrm{AxK}) & \vdash A \rightarrow(B \rightarrow A) \\ (\mathrm{AxB}) & \vdash(B \rightarrow C) \rightarrow((A \rightarrow B) \rightarrow(A \rightarrow C)) \\ (\mathrm{AxE}) & \vdash(A \rightarrow(B \rightarrow B)) \rightarrow(B \rightarrow(A \rightarrow A)) \\ (\mathrm{AxTO}) & \vdash((A \rightarrow B) \rightarrow(B \rightarrow A)) \rightarrow(B \rightarrow A) \\ (\mathrm{AxC}) & \vdash(A \rightarrow(B \rightarrow C)) \rightarrow(B \rightarrow(A \rightarrow C)) \\ (\mathrm{AxI}) & \vdash A \rightarrow A \\ (\mathrm{AxB}) & \vdash(A \rightarrow B) \rightarrow((B \rightarrow C) \rightarrow(A \rightarrow C)) \\ (\mathrm{Det}) & \text { From } \vdash A \rightarrow B \text { and } \vdash A, \text { infer } \vdash B \\ (\mathrm{Ax} \wedge \mathrm{E} 1) & \vdash(A \wedge B) \rightarrow A \\ (\mathrm{Ax} \wedge \mathrm{E} 2) & \vdash(A \wedge B) \rightarrow B \\ (\mathrm{Ax} \wedge \mathrm{I}) & \vdash((A \rightarrow B) \wedge(A \rightarrow C)) \rightarrow(A \rightarrow(B \wedge C)) \\ (\mathrm{Ax} \vee \mathrm{I} 1) & \vdash A \rightarrow(A \vee B) \\ (\mathrm{Ax} \vee \mathrm{I} 2) & \vdash B \rightarrow(A \vee B) \\ (\mathrm{Ax} \vee \mathrm{E}) & \vdash((A \rightarrow C) \wedge(B \rightarrow C)) \rightarrow((A \vee B) \rightarrow C) \\ (\mathrm{Adj}) & \text { From } \vdash A \text { and } \vdash B, \text { infer } \vdash A \wedge B\end{array}$

Specifically, Slaney's open questions involve the following three (background) positive logics.

1. $\mathrm{TW}^{+}[\mathrm{AxE}, \mathrm{AxTO}]$, the pure implicational fragment of which $\left(\mathrm{TW}^{\rightarrow}\right)$ is given by the axioms $\mathrm{AxB}, \mathrm{AxI}, \mathrm{AxB}^{\prime}, \mathrm{AxE}$, and $\mathrm{AxTO}$, and the rule Det. The full logic $\mathrm{TW}^{+}[\mathrm{AxE}, \mathrm{AxTO}]$ is then obtained by adding all of the axioms and rules for conjunction and disjunction to this implicational base. In other words, $\mathrm{TW}^{+}[\mathrm{AxE}, \mathrm{AxTO}]$ is given by: $\mathrm{AxB}$,

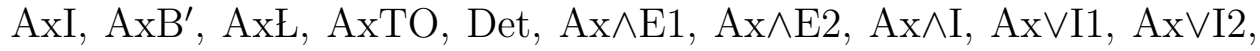
$A x \vee E$, and Adj.

2. $\mathrm{BCK}^{\rightarrow}[\mathrm{AxE}]$, which consists of the axioms $\mathrm{AxK}, \mathrm{AxB}, \mathrm{AxC}$, and AxE, and the rule Det.

\footnotetext{
${ }^{2}$ Here, I follow Slaney's [6] notation and nomenclature, which differs slightly from that of Harris \& Fitelson [1].
}

Australasian Journal of Logic (13:4) 2016, Article no. 1 
3. $\left.\mathrm{TW}^{\rightarrow}[\mathrm{Ax}\rfloor\right]$, which consists of the axioms $\mathrm{AxB}, \mathrm{AxI}, \mathrm{AxB}^{\prime}$, and AxE, and the rule Det.

\section{Four Other Principles Implicated in Slaney's Open Questions}

In addition to these three background positive logics, Slaney's open questions also involve the following four additional axioms/theorems and rules:

$$
\begin{array}{ll}
\text { (Dist) } & \vdash(A \wedge(B \vee C)) \rightarrow((A \wedge B) \vee(A \wedge C)) \\
\text { (RTO) } & \vdash(A \rightarrow B) \vee(B \rightarrow A) \\
\text { (IO) } & \vdash((A \rightarrow B) \rightarrow B)) \rightarrow(A \vee B) \\
\text { (Resid) } & \vdash(A \circ B) \rightarrow C \Longleftrightarrow \vdash A \rightarrow(B \rightarrow C)^{3}
\end{array}
$$

\section{Slaney's (Six) Open Questions and Their Solutions}

Slaney's first four open questions involve the background positive logic $\mathrm{TW}^{+}[\mathrm{AxE}$, AxTO]. The first two of these open questions are as follows.

1. Is (Dist) provable in $\mathrm{TW}^{+}[\mathrm{AxE}, \mathrm{AxTO}]$ ?

2. Is (RTO) provable in $\mathrm{TW}^{+}[\mathrm{AxE}, \mathrm{AxTO}]$ ?

Slaney [6, p. 65] notes that affirmative answers to both questions (1) and (2) are forthcoming, if it is possible to prove (IO) in $\mathrm{TW}^{+}$[AxE, AxTO]. Our first theorem therefore implies affirmative answers to both (1) and (2) ${ }_{4}^{4}$

Theorem 1. (IO) is provable in $T W^{+}[A x E, A x T O]$.

Slaney's next two open questions regarding $\mathrm{TW}^{+}[\mathrm{AxE}, \mathrm{AxTO}]$ involve the addition of a fusion operator ' $\circ$ ' to $\mathrm{TW}^{+}[\mathrm{AxE}, \mathrm{AxTO}]$, via the (Resid) rule.

\footnotetext{
${ }^{3}$ The meaning of " $p \Longleftrightarrow q$ " is "From $p$, infer $q$; and, from $q$, infer $p . "$ Thus, (Resid) adds a new "fusion" connective ' $\circ$,' which obeys the two-way rule of inference in question.

${ }^{4}$ See the ApPENDIX for Otter proofs of all theorems reported in this paper.
}

Australasian Journal of Logic (13:4) 2016, Article no. 1 
3. Is the addition of fusion a conservative extension of the positive logic $\mathrm{TW}^{+}[\mathrm{AxE}, \mathrm{AxTO}]$ ? That is, does the addition of (Resid) to $\mathrm{TW}^{+}[\mathrm{AxE}$, AxTO] imply no new theorems involving only $\langle\rightarrow, \wedge, \vee\rangle$ ?

4. If the answer to (3) is negative (i.e., if new $\langle\rightarrow, \wedge, \vee\rangle$-theorems are derivable upon adding (Resid) to $\left.\mathrm{TW}^{+}[\mathrm{AxE}, \mathrm{AxTO}]\right)$, then does the addition of (Resid) to $\mathrm{TW}^{+}[\mathrm{AxE}, \mathrm{AxTO}]$ allow us to prove both (AxK) and $(\mathrm{AxC})$ ?

Our second theorem implies both a negative answer to (3) and a positive answer to (4).

Theorem 2. (AxK) and $(A x C)$ are provable in $T W^{+}[A x E, A x T O]+($ Resid).

Slaney's fifth open question involves the background positive logic $\mathrm{BCK} \rightarrow[\mathrm{AxE}]$.

5. Is the addition of fusion, with its two-way rule (Resid), enough to generate a(nother) negation-free proof of $(\mathrm{AxTO})$ from $\mathrm{BCK}^{\rightarrow}[\mathrm{AxE}]$ ? In other words, is (AxTO) provable in $\mathrm{BCK}^{\rightarrow}[\mathrm{AxE}]+($ Resid $)$ ?

Our third theorem implies an affirmative answer to (5).

Theorem 3. (AxTO) is provable in $B C K^{\rightarrow}[A x E]+($ Resid).

That brings us to Slaney's sixth (and final) open question (implicitly asked on page 66), which involves his third background positive logic $\mathrm{TW}^{\rightarrow}[\mathrm{AxE}]$.

6. Is $(\mathrm{AxK})$ provable in $\mathrm{TW}^{\rightarrow}[\mathrm{AxE}]+($ Resid $)$ ?

Our fourth (and final) theorem implies an affirmative answer to (6).

Theorem 4. (AxK) is provable in $T W^{\rightarrow}[A x E]+$ (Resid).

Appendix: Proofs of Theorems

In this Appendix, I provide Otter proofs of our four theorems. Instead of using infix notation involving $\langle\rightarrow, \wedge, \vee, \circ\rangle$, I will use prefix notation involving $\langle i$, and, or, $f\rangle$. That is to say, we will adopt the following Otter notation:

$$
\vdash A \rightarrow B \mapsto \mathrm{p}(\mathrm{i}(\mathrm{A}, \mathrm{B}))
$$

Australasian Journal of Logic (13:4) 2016, Article no. 1 


$$
\begin{aligned}
& A \wedge B \mapsto \operatorname{and}(\mathrm{A}, \mathrm{B}) \\
& A \vee B \mapsto \operatorname{or}(\mathrm{A}, \mathrm{B}) \\
& A \circ B \mapsto \mathrm{f}(\mathrm{A}, \mathrm{B})
\end{aligned}
$$

See Harris \& Fitelson [1] for further explanation of how our Otter proof objects are to be interpreted (and related to more traditional presentations of proofs in sentential logics). The proofs presented here are the shortest/simplest proofs I was able to find using Otter.

Otter Proof of Theorem 15

Length of proof is 36 . Level of proof is 14 .

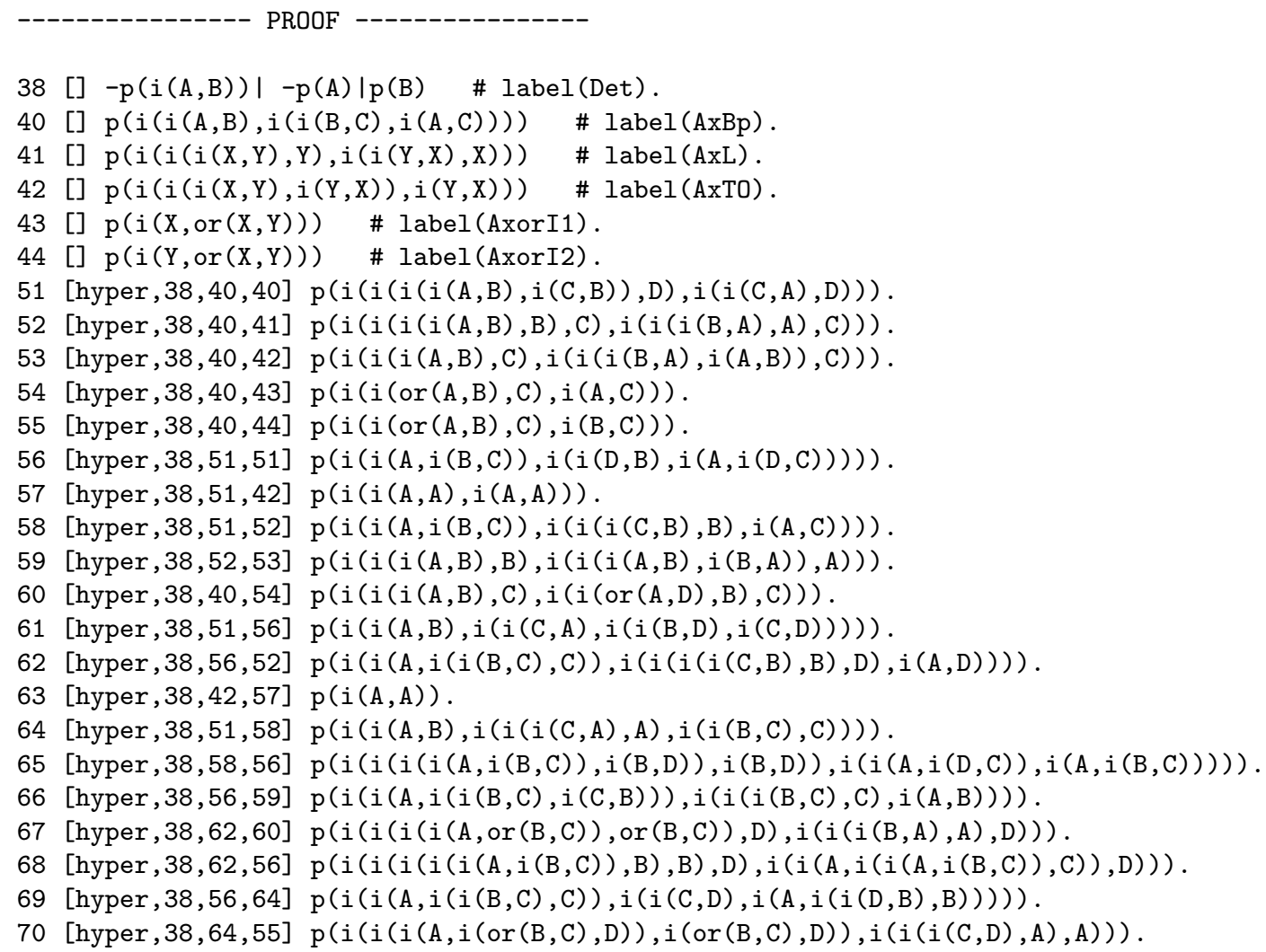

${ }^{5}$ In fact, this Otter proof establishes something stronger than Theorem 1. It shows that $(\mathrm{IO})$ is derivable from $\left\{\right.$ Det, $\left.\mathrm{AxB}^{\prime}, \mathrm{AXE}, \mathrm{AxTO}, \mathrm{Ax} \vee \mathrm{I} 1, \mathrm{Ax} \vee \mathrm{I} 2\right\}$. An Otter input file which verifies this proof is available from http://fitelson.org/slaney_theorem_1.in.

Australasian Journal of Logic (13:4) 2016, Article no. 1 


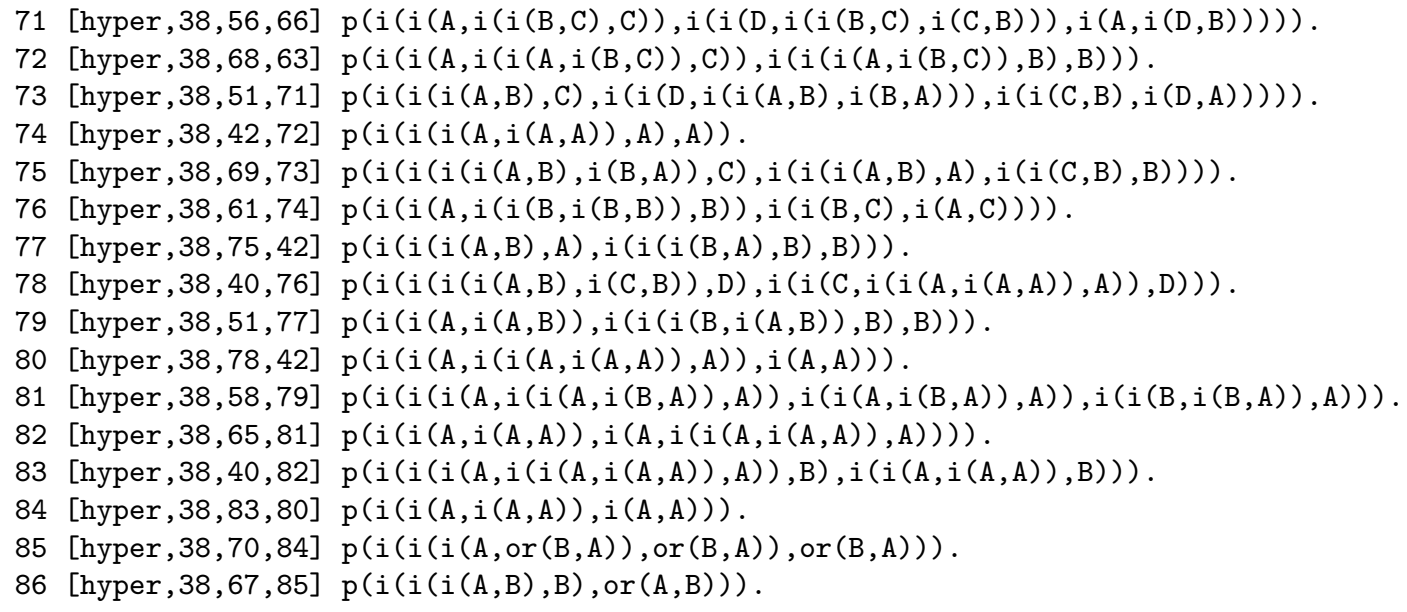
end of proof

Otter Proof of Theorem 2.6

Length of proof is 74 . Level of proof is 24 .

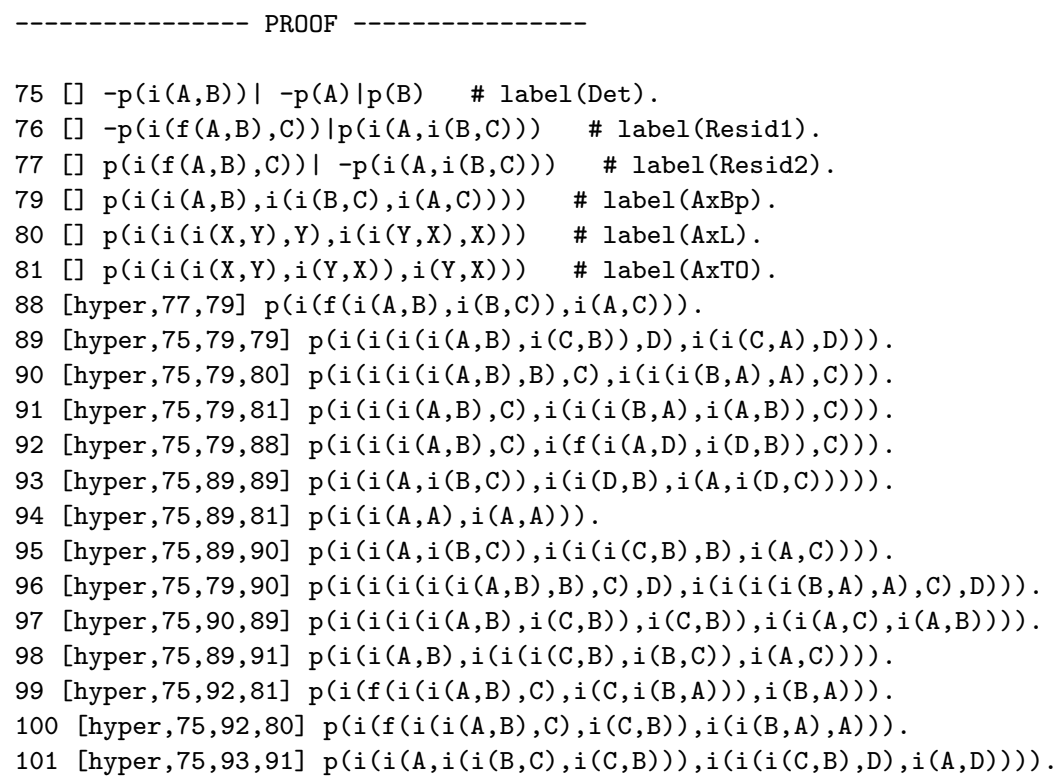

${ }^{6}$ In fact, this Otter proof establishes something stronger than Theorem 2. It shows that $(\mathrm{AxC})$ and $(\mathrm{AxK})$ are both derivable from \{Det, Resid, $\mathrm{AxB}^{\prime}, \mathrm{AxE}, \mathrm{AxTO}$. An Otter input file which verifies this proof is available from http://fitelson.org/slaney_ theorem_2.in.

Australasian Journal of Logic (13:4) 2016, Article no. 1 


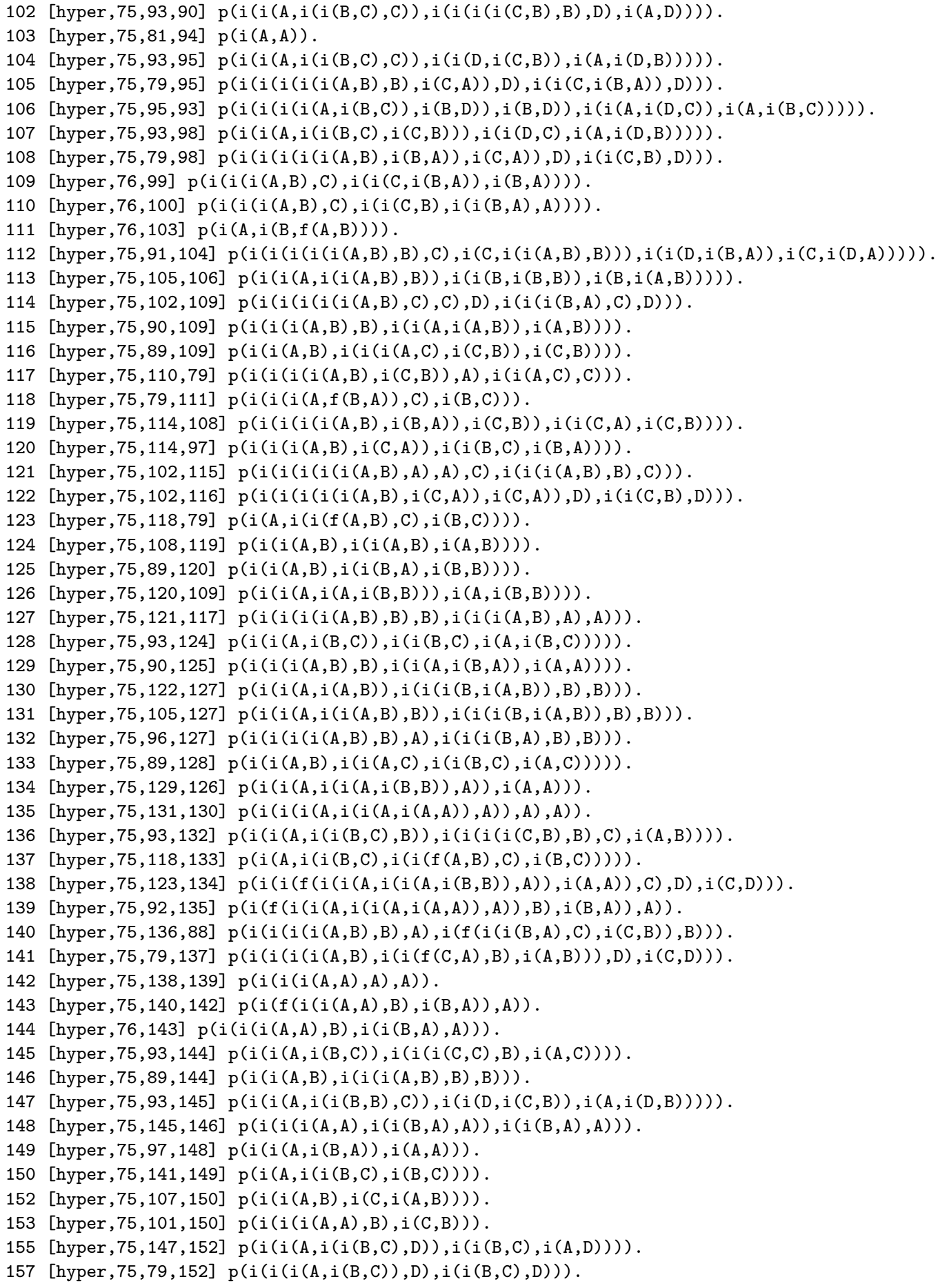

Australasian Journal of Logic (13:4) 2016, Article no. 1 


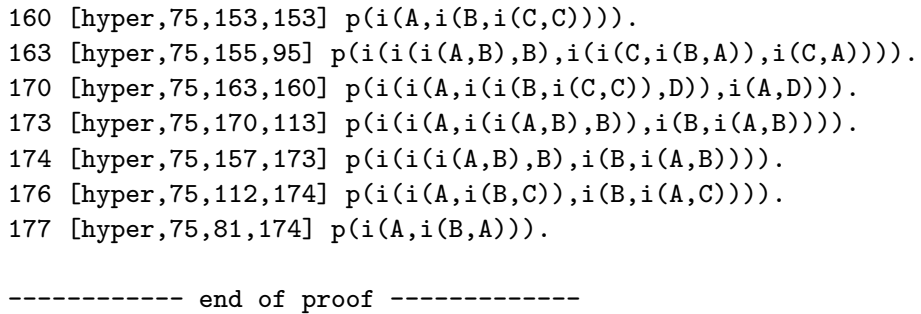

Otter Proof of Theorem 3.7

Length of proof is 28. Level of proof is 13 .

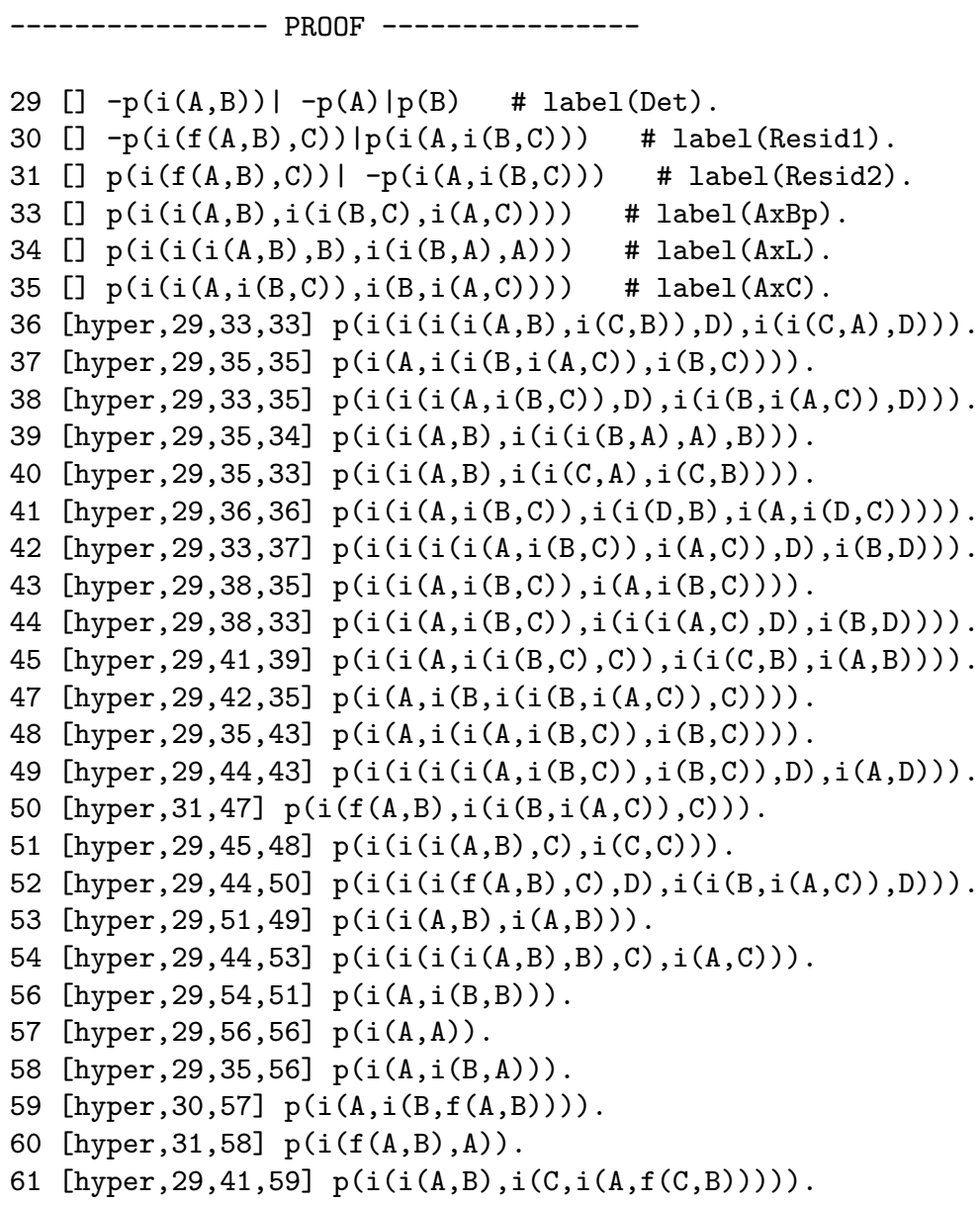

${ }^{7}$ In fact, this Otter proof establishes something stronger than Theorem 3. It shows that (AxTO) is derivable from $\left\{\right.$ Det, Resid, $\left.\mathrm{AxB}^{\prime}, \mathrm{AxC}, \mathrm{AxE}\right\}$. An Otter input file which verifies this proof is available from http://fitelson.org/slaney_theorem_3.in.

Australasian Journal of Logic (13:4) 2016, Article no. 1 
62 [hyper, 29,40,60] $p(i(i(A, f(B, C)), i(A, B)))$.

63 [hyper, 29,61,62] $p(i(A, i(i(B, f(C, D)), f(A, i(B, C)))))$.

64 [hyper, 29,45,63] $p(i(i(f(A, i(B, A)), B), i(A, B)))$.

65 [hyper, 29,52,64] $p(i(i(i(A, B), i(B, A)), i(B, A)))$.

end of proof

\section{Otter Proof of Theorem 48}

Length of proof is 52. Level of proof is 26 .

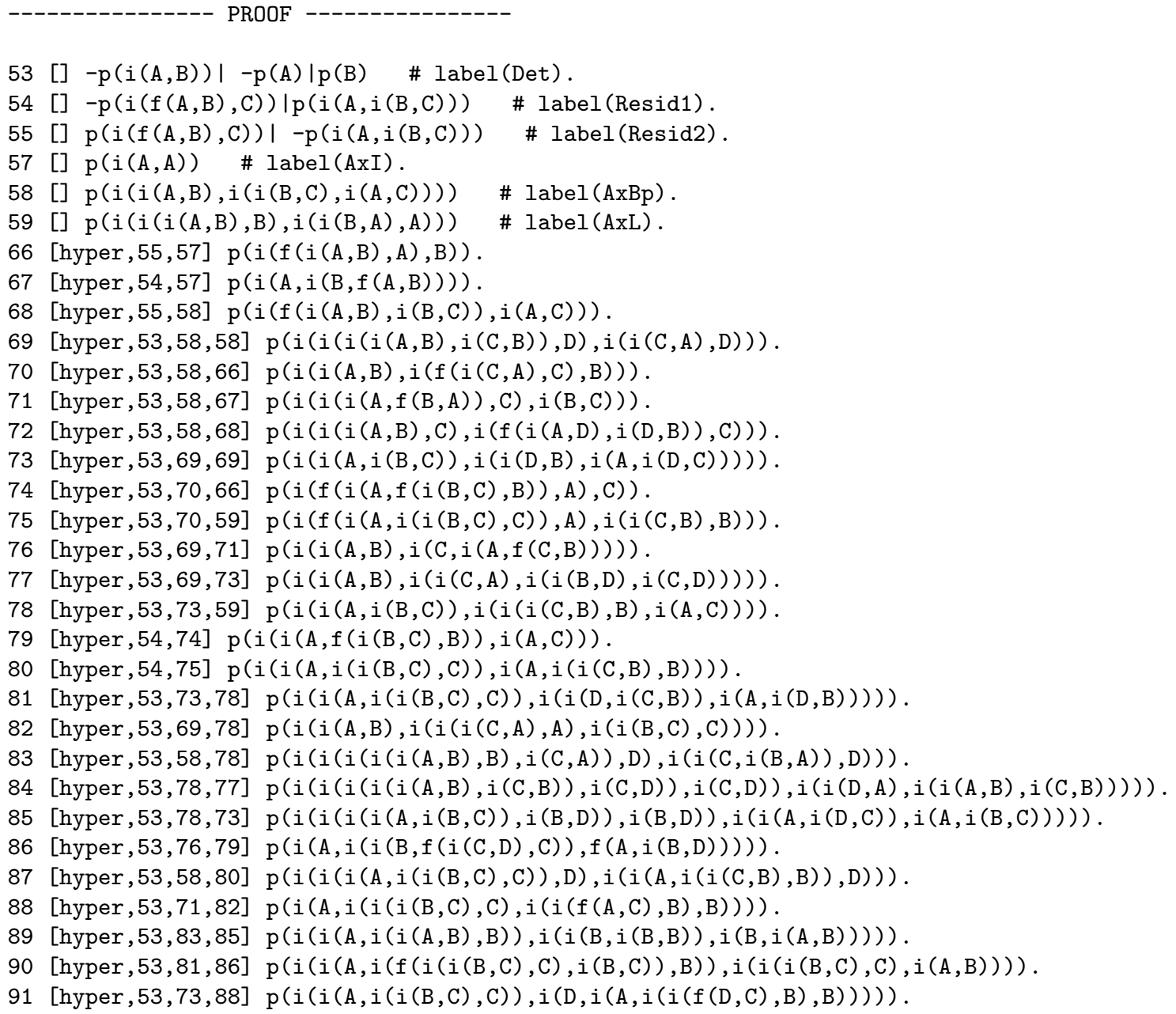

${ }^{8}$ In fact, this Otter proof establishes something stronger than Theorem 4. It shows that $(\mathrm{AxK})$ is derivable from \{Det, Resid, AxI, AxB', AxŁ\}. An Otter input file which verifies this proof is available from http://fitelson.org/slaney_theorem_4.in.

Australasian Journal of Logic (13:4) 2016, Article no. 1 


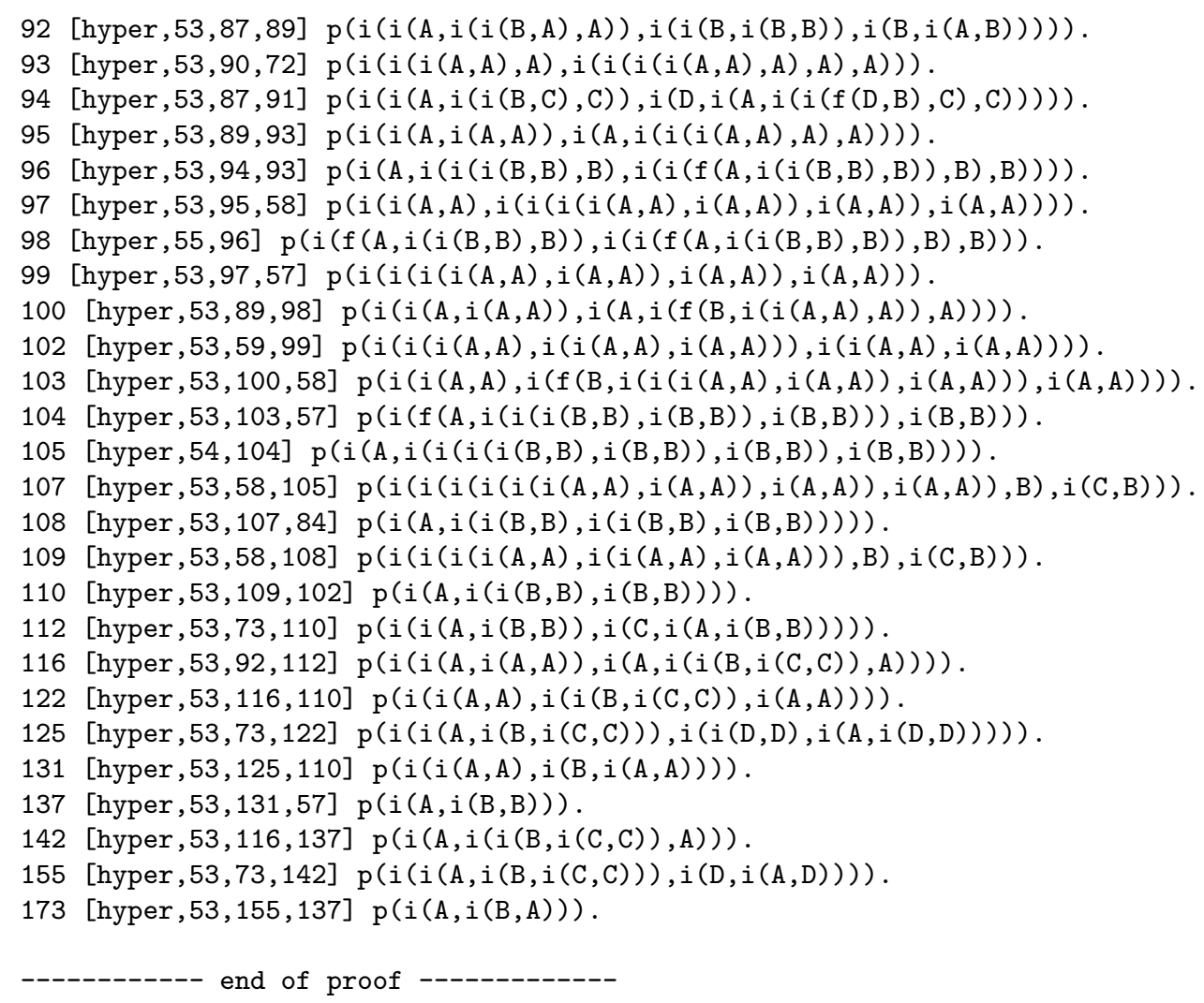

\section{References}

[1] Harris, K. and Fitelson, B.: Distributivity in $€_{\aleph_{0}}$ and Other Sentential Logics, Journal of Automated Reasoning, 27: 141-156 (2001).

[2] Kovacs, L. and Voronkov, A.: First order theorem proving and Vampire, in Sharygina, N., Veith, H. (eds.), Proceedings of the 25th International Conference on Computer-Aided Verification (CAV), volume 8044 of Lecture Notes in Computer Science, pp. 135. Springer (2013).

[3] McCune, W.: Prover9 and Mace4, available from http://www.cs.unm. edu/ mccune/prover9.

[4] McCune, W.: Otter 3.3 Reference Manual, Technical Report ANL/MSC-TM-263, Argonne National Laboratory, Argonne, IL, USA, 2003.

Australasian Journal of Logic (13:4) 2016, Article no. 1 
[5] Schulz, S.: System Description: E 1.8, in McMillan, K., Middeldorp, A. and Voronkov, A. (eds.), Logic for Programming, Artificial Intelligence, and Reasoning: 19th International Conference, LPAR-19, Stellenbosch, South Africa, December 14-19, 2013, Proceedings. Vol. 8312. Springer, 2013.

[6] Slaney, J.: More Proofs of an Axiom of Łukasiewicz, Journal of Automated Reasoning, 29: 59-66 (2002).

Australasian Journal of Logic (13:4) 2016, Article no. 1 